\title{
Electromagnetics Properties of Non-Relativistic Deuteron in Ground State
}

\author{
R. Yosi Aprian Sari and Denny Darmawan \\ Department of Physics Education, Yogyakarta State University, Indonesia \\ ryosia@yahoo.com
}

Received 26-01-2016, Revised 12-02-2016, Accepted 19-03-2016, Published 20-04-2016

\begin{abstract}
Proton-neutron interaction that makes up deuteron is a mixture state of ${ }^{3} S_{1}$ dan ${ }^{3} D_{1}$, which are each associated with a state $L=0, S=1$ and $L=2, S=1$. In the proton-neutron interaction, there are particle exchanges of medium range $(1 \mathrm{fm} \leq r \leq 2 \mathrm{fm})$ i.e. scalar meson exchange and the long range $(r>2 \mathrm{fm})$ i.e. one pion exchange. The electromagnetic properties of non-relativistic deuteron in the ground state can be found from the coupled differential equation, such as magnetic dipole moment, $\left\langle\mu_{\mathrm{D}}\right\rangle=0.856521 \mu_{N}$ and electrical quadrupole moment, $\left\langle Q_{\mathrm{D}}\right\rangle=0.00291396 \mathrm{~b}$.
\end{abstract}

Keywords: non-relativistic deuteron, mixture state, proton-neutron interaction, electromagnetic properties.

\section{INTRODUCTION}

Deuteron has one bound state resulted from the proton-neutron interaction passing through a particle exchange mechanism that was first introduced by Yukawa, 1935. Yukawa proposed that there are particles with mass parameter between the electron and nucleon mass which is responsible for the nuclear force. This particle is known as pion. Pion may be charged $\left(\pi^{+}, \pi^{-}\right)$or neutral $\left(\pi^{0}\right)$, the three form a triplet of isospin, $T=1$. Pion is member of a group of strongly interacting elementary particles (hadrons) which has medium mass, and are collectively called mesons; pion stands $\pi$-meson ${ }^{[1-4]}$.

According to Yukawa theory, every nucleon continuously emits and absorbs a pion; accompanying momentum transfer is equivalent to the action of force. Nuclear forces are repulsive at very short range as well as being attractive at far enough distance between nucleons, if this does not happen, the nucleon inside the nuclear will be bound together. This is the nuclear binding force. Potential for emission and absorption processes is one pion exchange potential, $V_{\mathrm{OPEP}^{[2]}}$.

Research by Friar, Gibson and Payne (FGP) obtained pionic effect of non-relativistic deuteron wave function using the OPE potential ${ }^{[5-6]}$. In their calculations, a pion-nucleon form factor replaces the high energy physics. The only influence of chiral symmetry in the FGP model is to require that the pion-nucleon coupling be in the $\tau \sigma . \nabla$ form $^{[7]}$.

In this paper, we develop what has been done by FGP, that is to determine the electromagnetic properties of non-relativistic deuteron in the bound state, namely the magnetic dipole moment and electric quadruple moment and distance interactions of protons and neutrons so that the resulting type of particles are exchanged.

Deuteron has unique property of having an abnormally low binding energy per nucleon, with no excited bound states ${ }^{[4]}$. In quantum mechanics, the two-particle potential $\widehat{V}$ is displayed as a matrix element between states of the two particle system presented by ket $\left|\hat{\vec{r}}_{1}, s_{1}, t_{1} ; \hat{\overrightarrow{\boldsymbol{r}}}_{2}, s_{2}, t_{2}\right\rangle$ with $s_{i}= \pm \frac{1}{2}$ and $t_{i}= \pm 1$, each of which is spin and isospin observable of $i$-th particle. The matrix elements take the form of: 


$$
\left\langle\hat{\overrightarrow{\boldsymbol{r}}}_{1}^{\prime}, S_{1}^{\prime}{ }_{1}, T^{\prime}{ }_{1} ; \hat{\overrightarrow{\boldsymbol{r}}}_{2}^{\prime}, S_{2}^{\prime}, T_{2}^{\prime}|\widehat{V}| \hat{\overrightarrow{\boldsymbol{r}}}_{1}, S_{1}, T_{1} ; \hat{\overrightarrow{\boldsymbol{r}}}_{2}, S_{2}, T_{2}\right\rangle
$$

where ket state of two particles, $\left|\widehat{\overrightarrow{\boldsymbol{r}}}_{1}, S_{1}, T_{1} ; \widehat{\overrightarrow{\boldsymbol{r}}}_{2}, S_{2}, T_{2}\right\rangle$ is the product of the wave function of spatial coordinates $\left(\left|\hat{\overrightarrow{\boldsymbol{r}}}_{1}\right\rangle,\left|\hat{\overrightarrow{\boldsymbol{r}}}_{2}\right\rangle\right)$, ket spin $\left(\left|S_{1}\right\rangle,\left|S_{2}\right\rangle\right)$ and isospin ket $\left(\left|T_{1}\right\rangle,\left|T_{2}\right\rangle\right)[8]$.

The wave function of the deuteron, $\psi_{\mathrm{D}}$ can be separated as the product of three parts of the wave function, which are intrinsic wave function of the proton, intrinsic wave functions of the neutron and the orbital wave function that describes the relative motion between the proton and the neutron. Since a proton and a neutron are just two different state of the nucleons, their intrinsic wave functions have the same parity, consequently, the product of their intrinsic wave functions has a positive parity that is not dependent on a single nucleon parity. Thus, parity of deuteron wave function is determined by relative motion between two nucleons.

Using symmetry considerations, the deuteron ground state has spin $S=1$ and isospin $T=$ 0 . There remain, however, two possibilities, $L=0$ and $L=2$, for the spatial part of the wave function. In spectroscopic notation, the $L=0, S=1$ state is represented as ${ }^{3} S_{1}$ (triplet- $S$ state) and the $L=2, S=1$ as ${ }^{3} D_{1}$ (triplet- $D$ state). There is, however, no fundamental reason to expect that this has to be true. In fact, we shall soon see that there is clear evidence that both the ${ }^{3} S_{1}$ - and the ${ }^{3} D_{1}$-components must be present in deuteron. This, in turn, leads to the conclusion that the nuclear force mixes different $L$-components in an eigenstate ${ }^{[2,9,10,11]}$.

By using common properties of symmetry or invariance of two nucleons, it can be shown that every eigenfunction has the spin-orbit and isospin parts, which can be described as the sum or a linear combination of the functions $\left|L S J M, T M_{T}\right\rangle$, where the sum is over $L$, consistent with the values of $J$ and parity of the eigenfunction. These functions are completely known: the isospin part labelled by $T M_{T}$ is the function $\rho_{M_{T}}^{T}\left(\tau_{1}, \tau_{2}\right)$, and the spin-angle functions labelled $L S J M$ are obtained by coupling the spherical harmonic $Y_{L M}(\theta, \phi)$ with the spin function $\chi^{S}\left(\sigma_{1}, \sigma_{2}\right)$.

For $S=0$ (spin-singlet), $L=J$ is the only possible value of $L$, whereas for $S=1$ (spintriplet), $L$ can have theree possible values: $J$ and $J \pm L$. The last two have parity opposite to that of $L=J$. Thus, the eigenfunction for $S=1$ and parity $\pi=-(-1)^{L}$ is the only complicated coupled wave function, it has the stucture

$$
\left.\frac{u}{r}\left|L=J-1, S=1, J M, T M_{T}\right\rangle+\frac{w}{r} L=J+1, S=1, J M, T M_{T}\right\rangle ;
$$

but the eigenfunction for $L=J(S=0$ atau $S=1)$ and parity $\pi=(-1)^{L}$ is uncoupled and has the simple expression

$$
\frac{u}{r}\left|L=J, S J M, T M_{T}\right\rangle
$$

The radial functions $u$ and $w$ are the only unknown parts of the two-nucleons wave function. Hence, the main purpose is to convert the Schrödinger equation into pure radial differential equations for $u$ and $w$. Obviously, (eq. 3) will yield an uncoupled equation for $u$, whereas (eq. 2) will give rise to coupled equation for $u$ and $w$. In order to eliminate the standard spin-angle and isospin parts of the wave functions from the Schrödinger equation, multiply the equation from the left by complex conjugate of these wave functions, integrate over the angles, and sum over the spin and isospin. This procedure gives rise to the matrix elements $\left\langle L^{\prime} S J M, T M_{T}|V| L S J M, T M_{T}\right\rangle$ in the potential term of the Schrödinger equation. 
Thus, the values of these matrix elements of the potential $\mathrm{V}$ can explicitly express the radial Schrödinger equation ${ }^{[11]}$.

The reduced mass of the two-nucleons system is $M^{*}$, where $\frac{1}{M^{*}}=\frac{1}{m_{p}}+\frac{1}{m_{n}} ; m_{p}$ and $m_{n}$ are proton and neutron mass, respectively. The Schrödinger equation in the centre-of-mass coordinate system is therefore given by

$$
\left(-\frac{\hbar^{2}}{M^{*}} \nabla^{2}+V\right) \Psi=E \Psi
$$

where the potential $V$ and the wavefunction $\Psi$ are functions of $r, \theta, \phi, \sigma_{1}, \sigma_{2}, \tau_{1}$ and $\tau_{2}$, and $E$ is energy in the centre-of-mass system. $E$ is negative ans equal to $-\epsilon$ ( $\epsilon$ is the binding energy of deuteron) when considered the bound-state problem.

By defining

$$
\left.\begin{array}{l}
v=-\frac{M^{*}}{\hbar^{2}} V \\
k^{2}=\frac{M^{*}}{\hbar^{2}} E \\
\gamma^{2}=\frac{M^{*}}{\hbar^{2}} \epsilon
\end{array}\right\}
$$

such that $k^{2}$ is positive for the scattering state and negative for the bound state, $\gamma^{2}$ is positive and $k=i \gamma$. Eigenfunctions $\Psi$ are labelled by the quantum number $S J M, T M_{T}{ }^{[11]}$.

To get the coupled radial equation in the Schrödinger equation

$$
\begin{gathered}
\frac{1}{r}\left[\left(\frac{d^{2}}{d r^{2}}-\frac{L^{2}}{r^{2}}\right)-v+k^{2}\right] \\
\times\left(u\left|L=J-1, S=1, J M, T M_{T}\right\rangle+w\left|L=J+1, S=1, J M, T M_{T}\right\rangle\right)=0
\end{gathered}
$$

where $L^{2}$ operate in angular momentum eigenfunction, to get the precise value $L(L+1)$. Multiply this equation from left, first by $\left\langle L=J-1, S=1, J M, T M_{T}\right|$ and second by $\left\langle L=J+1, S=1, J M, T M_{T}\right|$ and use the orthonormalization of this equation to get the simpler form gives two coupled radial equations

$$
\begin{aligned}
& {\left[\frac{d^{2}}{d r^{2}}-\frac{J(J+1)}{r^{2}}+k^{2}\right] u-\left\langle J-1, S=1, J M, T M_{T}|v| J-1, S=1, J M, T M_{T}\right\rangle u-} \\
& \left.J-1, S=1, J M, T M_{T}|v| J+1, S=1, J M, T M_{T}\right\rangle w=0 \\
& {\left[\frac{d^{2}}{d r^{2}}-\frac{(J+1)(J+2)}{r^{2}}+k^{2}\right] w-\left\langle J+1, S=1, J M, T M_{T}|v| J+1, S=1, J M, T M_{T}\right\rangle w-} \\
& \left.U+1, S=1, J M, T M_{T}|v| J-1, S=1, J M, T M_{T}\right\rangle u=0
\end{aligned}
$$

Matrix elements $v$ are the same for non-diagonal of $L$ since $\widehat{V}$ is hermitian. Radial function from non-diagonal matrix elements $v$ are stated as $\mathcal{H}(r)$. The same function from nondiagonal matrix elements $v$ of $L=J-1$ and $L=J+1$ state are stated as $\mathcal{F}(r)$ and $\mathcal{G}(r)$. Coupled radial equation can be written as 


$$
\frac{d^{2} u}{d r^{2}}-\frac{J(J-1)}{r^{2}} u+k^{2} u+\mathcal{F}(r) u+\mathcal{G}(r) w=0
$$

and

$$
\frac{d^{2} w}{d r^{2}}-\frac{(J+1)(J+2)}{r^{2}} w+k^{2} w+\mathcal{G}(r) w+\mathcal{H}(r) u=0
$$

A solution of $\left(\begin{array}{l}u \\ w\end{array}\right)$ from equation (4) can be multiplied by an arbitrary number, and $\left(\begin{array}{l}u \\ w\end{array}\right)$ is the solution for homogeneous equation. The potential, $u$ and $w$ are obtained at $r=r_{c}$ toward $r \rightarrow \infty$ [12].

The ground state of deuteron is a unique problem, that is its coupling, equation (8), with $k^{2}=-\gamma^{2}$ (bound state) and $J=1$. Wave function $u$ and $w$ each represents the radial function of ${ }^{3} S_{1}$ dan ${ }^{3} D_{1}$ state. The centrifugal form, $J(J-1)$ becomes zero in equation (8a). For $r \rightarrow \infty$, the asymptotic form $u / r$ and $w / r$ with $L=0$ and $L=2$, gives

$$
\begin{gathered}
u(r)=\mu r h_{0}^{(1)}(i \mu r)=\mu r\left[j_{0}(i \mu r)+i n_{0}(i \mu r)\right]=e^{-\mu r} ; \text { for } r \rightarrow \infty \\
w(r)=\mu r h_{2}^{(1)}(i \mu r)=e^{-\mu r}\left[1+\frac{3}{\mu r}+\frac{3}{(\mu r)^{2}}\right] ; \text { for } r \rightarrow \infty
\end{gathered}
$$

The coupled equation from equation (8) can be written in a simpler form,

$$
\begin{aligned}
& \frac{d^{2} u}{d r^{2}}=\bar{e} u+\bar{f} w \\
& \frac{d^{2} w}{d r^{2}}=\bar{g} w+\bar{h} u
\end{aligned}
$$

where $\bar{e}, \bar{f}, \bar{g}$ and $\bar{h}$ as function of $r, \bar{e}=\left[\frac{J(J-1)}{r^{2}}-k^{2}-\mathcal{F}(r)\right], \bar{f}=-\mathcal{G}(r), \bar{g}=$ $\left[\frac{(J+1)(J+2)}{r^{2}}-k^{2}-\mathcal{G}(r)\right]$, and $\bar{h}=-\mathcal{H}(r)$, with normalization condition ${ }^{[6,7,12]}$,

$$
\int_{0}^{\infty}\left[u^{2}(r)+w^{2}(r)\right] d r=1
$$

\section{METHOD}

Both of the coupled differential equations (eq. 10) can not be solved analytically, and can only be solved through numerical computation, i.e. boundary value problem method. These equations can be written in the form of eigenfunction equation,

$$
\left(\begin{array}{ll}
M_{1} & -C \\
-C & M_{2}
\end{array}\right)\left(\begin{array}{l}
\Phi_{1} \\
\Phi_{2}
\end{array}\right)=\lambda\left(\begin{array}{l}
\Phi_{1} \\
\Phi_{2}
\end{array}\right)
$$

where $\Phi_{1}$ and $\Phi_{2}$ are set values of $u(r)$ and $w(r)$, respectively, and $\lambda$ is eigenvalue of energy $E$.

For the state $|L S ; J M\rangle$, expectation value of magnetic dipole moment of deuteron has to be evaluated for $M=J$,

$$
\begin{aligned}
& \left\langle L S J M=J\left|\mu_{z}\right| L S J M=J\right\rangle \\
& =\frac{1}{2(J+1)}\left[\left(\mu_{\mathrm{p}}+\mu_{\mathrm{n}}+\frac{1}{2}\right) J(J+1)+\left(\mu_{\mathrm{p}}+\mu_{\mathrm{n}}-\frac{1}{2}\right)(S(S+1)-L(L+1))\right]
\end{aligned}
$$

For deuteron ${ }^{3} S_{1}$-state, $L=0$, and the expectation value of the magnetic dipole operator reduces to a sum of intrinsic dipole moments of a proton and a neutron,

$$
\mu_{\mathrm{D}}\left({ }^{3} S_{1}\right)=\mu_{\mathrm{p}}+\mu_{\mathrm{n}}=0.879805 \mu_{\mathrm{N}}
$$


where $\mu_{\mathrm{p}}$ and $\mu_{\mathrm{n}}$ are the dipole moments of the proton and neutron, respectively.

The electrical quadrupole moment of a nuclear state is defined as expectation value of $Q_{0}$ in the substate of maximum $M$,

$$
Q_{A}=\left\langle J M=J\left|Q_{0}\right| J M=J\right\rangle
$$

in the same way as the definition of magnetic dipole moment.

From the point of view of angular momentum, any nuclear state with $J<1$ cannot have a quadrupole moment different from zero. The expectation value $\left\langle J M\left|Q_{0}\right| J M\right\rangle$ vanishes if the three angular momenta involved, $J, 2, J$, cannot be coupled together to form closed triangle. At the time, since $Q_{0}$ operates only in the coordinates space, it is independent of the total intrinsic spin $S$. This means that the orbital angular momentum $L$ of the state must also be greater or equal to 1 . For this reason, the expectation value of $Q_{0}$ vanishes in the ${ }^{3} S_{1}$-state; the existence of a nonvanishing quadrupole moment is therefore a direct evidence of the presence of ${ }^{3} D_{1}$-component in the deuteron ground state ${ }^{[2,11]}$.

The connection between the spatial part of the wave function and the quadrupole moment, assuming for the time being that the deuteron is in a state of definite orbital angular momentum $L$. Such a wave function $|L S ; J M\rangle$ can be represented by the product of spatial part $\left|L M_{L}\right\rangle$ and intrinsic spin part $\left|S M_{S}\right\rangle$, coupled together to total angular momentum $(J, M)$,

$$
|L S ; J M\rangle=\sum_{M_{L} M_{S}}\left\langle L M_{L} S M_{S} \mid J M\right\rangle\left|L M_{L}\right\rangle\left|S M_{S}\right\rangle
$$

where $\left\langle L M_{L} S M_{S} \mid J M\right\rangle\left|L M_{L}\right\rangle\left|S M_{S}\right\rangle$ is the Clebsch-Gordan coefficient. The expectation value of electric quadrupole moment

$$
\begin{aligned}
& \left\langle Q_{\mathrm{D}}(L)\right\rangle=\left\langle L S ; J M\left|Q_{0}\right| L S ; J M\right\rangle \\
& =\sum_{M_{L} M_{S}} \sum_{M_{L}^{\prime} M_{S}^{\prime}}\left\langle L M_{L} S M_{S} \mid J M\right\rangle\left\langle L M^{\prime}{ }_{L} S M^{\prime}{ }_{S} \mid J M\right\rangle\left\langle L M_{L} S M_{S}\left|Q_{0}\right| L M^{\prime}{ }_{L} S M^{\prime}{ }_{S}\right\rangle
\end{aligned}
$$

For more realistic model, a linear combination of ${ }^{3} S_{1}$ - and ${ }^{3} D_{1}$-components have been done earlier in Eq. (15) for the magnetic dipole moment. The deuteron electric quadrupole moment now has the form

$$
\begin{aligned}
& \left\langle Q_{\mathrm{D}}\right\rangle=a^{2}\left\langle{ }^{3} S_{1} M=1\left|Q_{0}\right|{ }^{3} S_{1} M=1\right\rangle \\
& +b^{2}\left\langle{ }^{3} D_{1} M=1\left|Q_{0}\right|{ }^{3} D_{1} M=1\right\rangle+2 a b\left\langle{ }^{3} S_{1} M=1\left|Q_{0}\right|{ }^{3} D_{1} M=1\right\rangle
\end{aligned}
$$

The first term vanishes, since $L=0$, the main contribution is likely to come from the last term, since ${ }^{3} D_{1}$-component is only a few percent of the total and $|a|>|b|$ as a result. This term involves an off-diagonal matrix element and depends on the radial integral [2],

$$
\left\langle{ }^{3} S_{1} M=1\left|Q_{0}\right|^{3} D_{1} M=1\right\rangle \propto \int_{0}^{\infty} R_{S}^{*}(r) R_{D}(r) r^{2} d r
$$

\section{RESULTS AND DISCUSSION}

The calculated wave functions of $u(r)$ and $w(r)$ are plotted in Figure 1. The calculated binding energy $E$ is $=-2.2427356 \mathrm{MeV}^{[4,6]}$. 


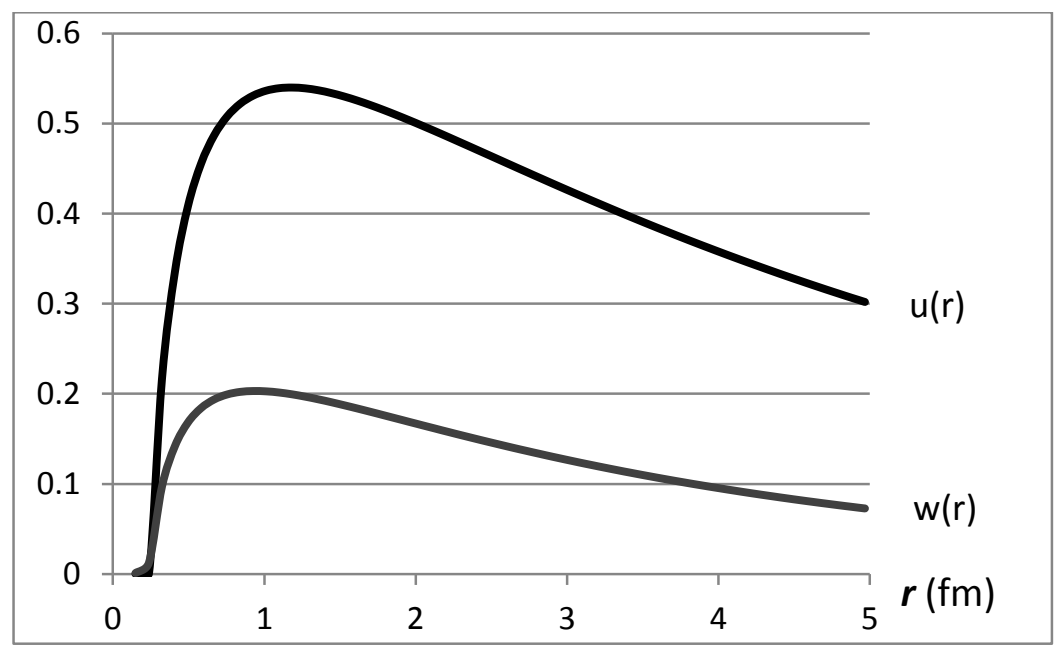

Figure 1. The non-relativistic deuteron wave function in the ground state

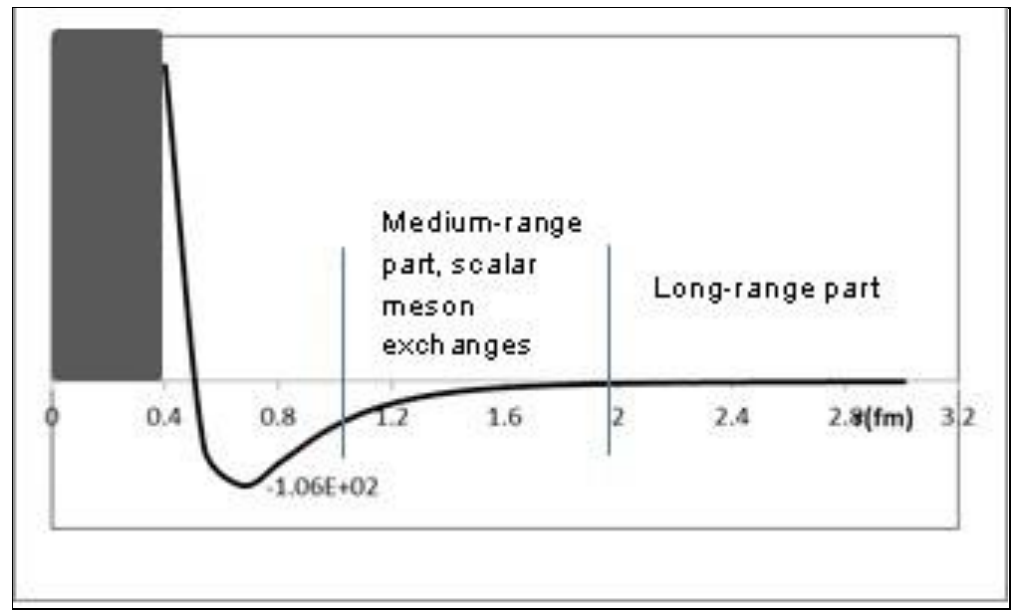

Figure 2. Proton and Neutron Interaction Potential

From computation, it is known that there is a repulsive force starting from $r_{c}=0,4 \mathrm{fm}$, meaning that $0 \leq r \leq r_{c}$ region is an infinite height potential wall, thus the probability to find particles (deuteron) in this interval is zero (see figure 1 and 2$)^{[12]}$.

For far enough distance, $r \rightarrow \infty$, there is an attractive force so that both particles move closer. The main part of the attractive force is located around radius of $1 \sim 2 \mathrm{fm}$, where the scalar meson $(\pi, \rho, \omega, \sigma)$ exchange takes place, and $r>2 \mathrm{fm}$ for the pion exchange (see figure 2).

The final result of Eq. (8) is almost the same as the experimental value of $0.856521 \mu_{N}$. The small difference,

$$
\mu_{\mathrm{D}}-\mu_{\mathrm{D}}\left({ }^{3} S_{1}\right)=0.856521-0.879805=-0.023284 \mu_{\mathrm{N}}
$$

is, however, worth more careful consideration. Small differences in this expectation value caused by contribution from charged (virtual) mesons exchange between the proton and the neutron, and this has not been included in Eq. (12) and there is a small admixture of the ${ }^{3} D_{1}$-state in the deuteron ground state ${ }^{[4,10,11]}$.

It is a great interest to know the amount of $D$-state admixture in the deuteron wave function. This can be quantified by the $D$-state probability ${ }^{[13-14]}$ : 


$$
P_{D}=\int_{0}^{\infty} w^{2}(r) d r=0.0406=4.06 \%
$$

For a linear combination of ${ }^{3} S_{1}$ - and ${ }^{3} D_{1}$-components, the deuteron wave function can be written as

$$
\left|\mu_{\mathrm{D}}\right\rangle=a\left|{ }^{3} S_{1}\right\rangle+b\left|{ }^{3} D_{1}\right\rangle
$$

with the normalization condition ${ }^{[2,4,10,11,13]}$.

$$
a^{2}+b^{2}=1
$$

The first term vanishes, since $L=0$. The main contribution is likely to come from the last term, as the ${ }^{3} D_{1}$-component is only a few percent of the total and $|a|>|b|$ as a result $[4,10,11,13]$. So that the contribution of quadrupole moment of deuteron can be written as

$$
Q_{\mathrm{D}}=\frac{1}{20} \int_{0}^{\infty} r^{2} w(r)(2 \sqrt{2} u(r)-w(r)) d r
$$

The above equation explicitly shows that the quadrupole moment does not vanish and require mixing with a state- $D$, the components $w(r)$, and assuming the charge centered on nucleons. $D$-state contribution to the deuteron quadrupole moment $\left(Q_{\mathrm{D}}=\right.$ $0.002913967 \mathrm{~b}$ ), in the shape of flat spheroid (oblate) $)^{[12-13]}$.

\section{CONCLUSION}

The deuteron ground state has $S=1$ and $T=0$. There remains, however, two possibilities, $L=0$ and $L=2$, for the spatial part of the wave function. In spectroscopic notation, the $L=0, S=1$ state is represented as ${ }^{3} S_{1}$ (triplet- $S$ state) and the $L=2, S=1$ as ${ }^{3} D_{1}$ (triplet- $D$ state). There is a small admixture of the ${ }^{3} D_{1}$-state $(\sim 4 \%)$ in the ground state of the deuteron. The electromagnetic properties of non-relativistic deuteron wave function in the ground state can be derived from the mixture state, giving dipole magnetic moment, $\left\langle\mu_{\mathrm{D}}\right\rangle=0.856521 \mu_{N}$ and quadrupole electric moment, $\left\langle Q_{\mathrm{D}}\right\rangle=0.002913967 \mathrm{~b}$. From the calculation, in short distance $\left(r_{\mathrm{c}}=0,4 \mathrm{fm}\right)$ there is an infinite potential wall, thus there is no deuteron exist in the interval of $\left(0 \leq r \leq r_{\mathrm{c}}\right) \mathrm{fm}$. In medium distance $(1 \leq r \leq 2) \mathrm{fm}$, there is a scalar meson $(\pi, \rho, \omega, \sigma)$ exchange, and in long distance $(r>2 \mathrm{fm})$ there is a single pion exchange.

\section{ACKNOWLEDGEMENTS}

This research was supported by Hibah Bersaing Research, Ministry of Research, Technology and Higher Education, contract number: 20/Hibah Bersaing/UN.34.21/2015, March 2, 2015.

\section{REFERENCES}

1. Rho, M., and Wilkinson, D. 1979. Mesons in Nuclei. North-Holland Publishing Company, Amsterdam, Netherlands.

2. Wong, S.S.M. 1990. Introductory Nuclear Physics. Prentice Hall: New Jersey.

3. Eisenberg, J.M., and W. Greiner. 1986. Nuclear Theory: Microscopic Theory of the Nucleus. North-Holland Publishing Company, Amsterdam, Netherlands.

4. R. Yosi Aprian Sari., Supardi., Agung BSU., dan Hermanto, A. 2012. Dinamika Pertukaran Partikel Pada Interaksi Nukleon-Nukleon dalam Potensial Lokal. Journal Indonesian Journal of Applied Physics (IJAP), Vol. 02, No. 1. ISSN: 2089-0133, 
http://ijap.mipa.uns.ac.id

5. Friar, J.L., Gibson, B. F., and Payne, G. L. 1984. Phys. Rev. C30, 1084

6. Ballot, J.L. and Robilotta, M. R. 1992. Phys. Rev.C 45, 986

7. Cooke, J.R. and Miller, G. A. Phys.Rev.C65 067001 (2002)

8. Ring, P., and Schuck, P. 1980. The Nuclear Many-Body Problem. Springer-Verlag: Berlin Heidelberg, Germany.

9. Sprung, D.W.L., W. van Dijk, E. Wang, D. C. Zheng, P. Sarriguren, and J. Martorell, Phys. Rev. C49, 2942 (1994)

10. Gilman, R and Gross, F. 2002. J.Phys. G28 R37-R116

11. Pal, M. K. 1982. Theory of Nuclear Stucture. Affiliated East-West Press PVT. LTD, New Delhi

12. Valderraman, M. P. and Arriola. E. R. 2005. Phys.Rev. C72:054002

13. Forest, J.L. 2000. Effects of Nonlocal One-Pion-Exchange Potential in Deuteron, Phys. Rev C61, 034007

14. R. Yosi Aprian Sari, Denny Darmawan (2015) "Non-Relativistic Deuteron In One-Pion Exchange Potential" Proceeding Internasional Symposium; The Application of Nuclear Technology to Support National Sustainable Development: Health, Agriculture, Energy, Industry and Environment; October 26-28, 2015, Salatiga 